\title{
$\mathrm{Al}^{3+}$ 可视化水溶性分子荧光探针的合成及其作用研究
}

\author{
马 拓 张 瑾刘龙珠 贺云张尊听*
}

(教育部药用资源与天然药物化学重点实验室 西北濒危药材资源开发国家工程实验室

陕西师范大学化学化工学院 西安 710062)

\begin{abstract}
摘要 设计并合成了 3 种嘧啶并 $[1,6-a$ 苯并咪唑磺化物: 4-(2'-差基苯基)嘧啶并 $[1,6-a]$ 苯并咪唑-5'-磺酸钠(L1)、4-(2'-差年

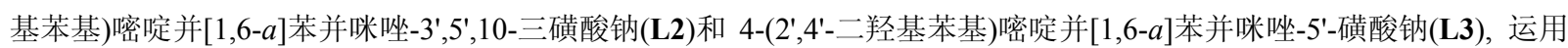
质谱和核磁等方法对其结构进行表征. 运用荧光光谱法考察了 $\mathbf{L 1}, \mathbf{L 2}$ 和 $\mathbf{L 3}$ 对 $\mathrm{Al}^{3+}$ 的识别作用, 结果表明: 在水溶液中 $\mathbf{L 2}$ 能与 $\mathrm{Al}^{3+}$ 配合, 配合后的荧光光谱强度增强, 而加入其它金属离子 $\left(\mathrm{Cu}^{2+}, \mathrm{Hg}^{2+}, \mathrm{K}^{+}, \mathrm{Mg}^{2+}, \mathrm{Na}^{+}, \mathrm{Ni}^{2+}, \mathrm{Pb}^{2+}, \mathrm{Zn}^{2+}\right.$, $\mathrm{Cr}^{3+}, \mathrm{Fe}^{3+}$ )基本没有变化, 表明 $\mathbf{L} 2$ 对 $\mathrm{Al}^{3+}$ 具有专一的识别性. 通过 Job's plot 曲线和荧光滴定实验确定 $\mathbf{L 2}$ 和 $\mathrm{Al}^{3+}$ 以 1 : 2 的比例进行配合, $\mathbf{L} 2$ 对 $\mathrm{Al}^{3+}$ 的检出限为 $0.073 \mu \mathrm{mol} / \mathrm{L}$, 并且 $\mathbf{L} 2$ 能够可视化检测 $\mathrm{Al}^{3+}$.
\end{abstract}

关键词＼cjkstart配位; 铝离子; 苂光探针; 激发态内分子质子转移; 可视化检测

\section{Synthesis and Characterization of a Visualization and Water-Soluble Fluorescent Probe for $\mathrm{Al}^{3+}$}

\author{
Ma, Tuo Zhang, Jin Liu, Longzhu He, Yun Zhang, Zunting* \\ (Key Laboratory of the Ministry of Education for Medicinal Resources and Natural Pharmaceutical Chemistry, National En- \\ gineering Laboratory for Resource Development of Endangered Crude Drugs in Northwest of China and \\ School of Chemistry and Chemical Engineering, Shaanxi Normal University, Xi'an 710062)
}

\begin{abstract}
In order to improve the water-solubility of 4-(2'-hydroxyphenyl)-pyrimido[1,6- $a]$ benzimidazole and 4-(2'-hydrxyphenyl-4'-isopropoxy)-pyrimido[1,6- $a$ ] benzimidazoles, they were sulfonated in $98 \% \mathrm{H}_{2} \mathrm{SO}_{4}$. Sodium 4-(2'-hydroxyphenyl)pyrimido[1,6- $a$ ] benzimidazole-5-sulfonate (L1), sodium 4-(2'-hydroxyphenyl)-pyrimido[1,6- $a$ ]benzimidazole-3',5',10'-threesulfonate (L2), sodium 4-(2',4'-dihydroxyphenyl)pyrimido[1,6- $a$ ]benzimidazol-5-sulfonate (L3) were obtained and indentified by the method of NMR, IR and MS. Fluorescence spectra of L1, L2 and L3 were detected, and they have the ESIPT effect with a large Stokes shift $(175 \sim 225 \mathrm{~nm})$. The structures of these compounds could be complex with $\mathrm{Al}^{3+}$, and the fluorescence spectra of $\mathrm{Al}^{3+}$ in $\mathbf{L 1}, \mathbf{L 2}$ and $\mathbf{L 3}$ were analyzed. The results showed that $\mathbf{L 2}$ exhibited good sensitivity selectivity to $\mathrm{Al}^{3+}$. The fluorescence of $\mathbf{L 2}$ was strengthened dramatically in the presence of $\mathrm{Al}^{3+}$. Other metal ions, such as $\mathrm{Cu}^{2+}, \mathrm{Hg}^{2+}, \mathrm{K}^{+}$, $\mathrm{Mg}^{2+}, \mathrm{Na}^{+}, \mathrm{Ni}^{2+}, \mathrm{Pb}^{2+}, \mathrm{Zn}^{2+}, \mathrm{Cr}^{3+}, \mathrm{Fe}^{3+}$ induced negligible fluorescence strengthening for $\mathbf{L} 2$ under the same conditions. When the concentration $(0 \sim 20 \mu \mathrm{mol} / \mathrm{L})$ of $\mathrm{Al}^{3+}$ was increased, the fluorescence intensity of $\mathbf{L} \mathbf{2}(10 \mu \mathrm{mol} / \mathrm{L})$ was also increased. The Job's plot and fluorescence titration indicated that the stoichiometry of $\mathbf{L} 2-\mathrm{Al}^{3+}$ was $1: 2$ and the detection limit of $\mathrm{Al}^{3+}$ was $0.073 \mu \mathrm{mol} / \mathrm{L}$. Moreover, $\mathbf{L} 2$ was used for the visual detection of $\mathrm{Al}^{3+}$.
\end{abstract}

Keywords coordinate; aluminum ions; fluorescent probe; excited state intramolecular proton transfer (ESIPT); visual detection

在岩石圈中铝是排行第三丰富的元素, 占地球质量 约 $8 \%$. 有着许多不寻常的性质, 因此被广泛应用于各个 领域中 ${ }^{[1,2]}$, 并且可在鱼、藻类、细菌和其他物种中逐级 传递 ${ }^{[3]}$. 铝的离子状态 $\left(\mathrm{Al}^{3+}\right)$ 具有改变生物反应的能
力, 从而影响或抑制它们的某些功能. 铝离子对生物的 神经系统具有毒害作用, 它会影响神经纤维酶和神经递 质的传递. 铝离子的毒性被认为是神经性疾病的主要原 因，例如帕金森氏病、阿尔海默氏病和透析性脑病 ${ }^{[6 ~ 9]}$.

\footnotetext{
*E-mail: zhangzt@snnu.edu.cn

Received May 8, 2014; revised May 24, 2014; published online June 3, 2014.

Project supported by the National Natural Science Foundation of China (No. 21372150), and the Fundamental Research Funds for the Central Universities (No. GK261001095).

国家自然科学基金(No. 21372150)、高校基本科研业务费专项资金(No. GK261001095)资助项目.
} 
人类每周对铝的膳食量上限为 $7 \mathrm{mg} / \mathrm{kg}$ 体重. 研究表明, 若过多的摄入铝会影响神经系统的信号传递, 进而对人 的记忆力造成不同程度影响. 荧光分析法是一种先进的 分析方法, 较其它方法 (电子探针法、质谱法和光谱法) 具有很多的优点, 如灵敏度高、选择性强和响应速度快 ${ }^{[10]}$, 可以广泛地应用于一些金属离子和阴离子的检测. 因而对于铝离子具有高选择性和灵敏性的荧光探针的 研发具有重要的意义.

激发态内分子质子转移(Excited state intramolecular proton transfer, ESIPT)是指一些有机化合物在受到光、 热、电等激发后，基态分子吸收能量跃迁至激发态时， 分子中的质子 (如羟基或者氨基)通过氢键转移到分子内 与其较近的杂原子上(如: 氮、氧或者硫), 从而形成互变 异构体的过程 ${ }^{111}$. 基于该原理, 人们设计并合成了一系 列荧光分子探针 ${ }^{[12 \sim 14]}$.

迄今为止人们已经设计了许多可用于 $\mathrm{Al}^{3+}$ 检测的 分子荧光探针, 但是大多数都是在有机溶剂中进行检 测, 水溶性的分子荧光探针相对比较少(Scheme 1) ${ }^{[15,16]}$.
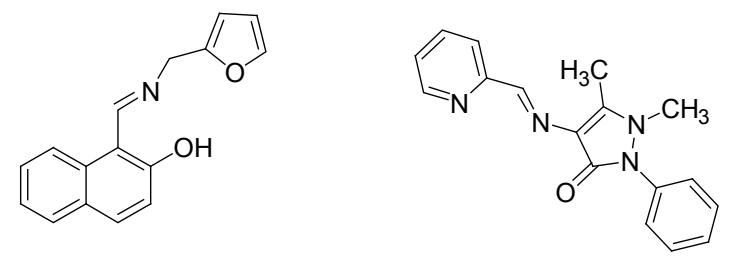

\section{Scheme 1}

本文以取代 3-( $N, N$-二甲基氨基)-1-(2'-羟基苯基)-2烯丙酮和 2-氨基苯并咪唑为原料合成了嘧啶并 $[1,6-a$ ]苯 并咪唑化合物 ${ }^{[17]}$, 通过磺化反应合成了三种嘧啶并 [1,6- $a$ ]苯并咪唑磺化物：4-(2'-差基苯基)嘧啶并 $[1,6-a$ 苯 并咪唑-5'-磺酸钠 (L1)、4-(2'-羟基苯基)嘧啶并 $[1,6-a]$ 苯 并咪唑-3',5',10-三磺酸钠(L2) 和 4-(2',4'二垟基苯基)嘧啶 并 $[1,6-a]$ 苯并咪唑-5'-磺酸钠 $(\mathbf{L} 3)$ (Scheme 2). 采用荧光 光谱法研究了 $\mathrm{L1}, \mathrm{L2}$ 和 $\mathrm{L3}$ 对 $\mathrm{Al}^{3+}$ 的识别作用. 结果表 明 $\mathbf{L 2}$ 对 $\mathrm{Al}^{3+}$ 具有很好的络合能力, 并且具有很高的灵 敏度和很好的选择性, 检出限达 $0.073 \mu \mathrm{mol} / \mathrm{L}$. 通过荧 光滴定、Job's plot 曲线和核磁滴定确定了 $\mathbf{L 2}$ 和 $\mathrm{Al}^{3+}$ 的 配位比为 $1: 2 . \mathbf{L 2}$ 对 $\mathrm{Al}^{3+}$ 具有专一识别性, 在 $\mathrm{Cu}^{2+}$, $\mathrm{Hg}^{2+}, \mathrm{K}^{+}, \mathrm{Mg}^{2}, \mathrm{Na}^{+}, \mathrm{Ni}^{2+}, \mathrm{Pb}^{2+}, \mathrm{Zn}^{2+}, \mathrm{Cr}^{3+}, \mathrm{Fe}^{3+}$ 的存在 下, 仍能对 $\mathrm{Al}^{3+}$ 进行识别, 表现出较强的抗干扰作用. $\mathbf{L 2}$ 能够可视化检测 $\mathrm{Al}^{3+}$, 在 $365 \mathrm{~nm}$ 紫外灯下激发, 可 以发出绿色荧光.

\section{1 结果与讨论}

\section{1 合成部分}

参考文献[17]合成了嘧啶并[1,6- $a$ ]苯并咪唑化合物

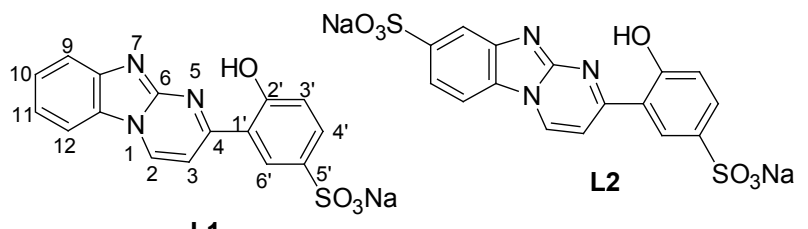

L1

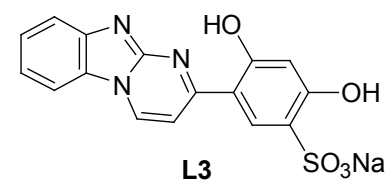

Scheme 2

(1 和 2), 它们都具有 ESIPT 效应, 并且由于 2 '位羟基和 5 位氮原子的存在使其能与金属离子配体. 为了增加水 溶性对 1 和 2 进行磺化合成了 L1, L2 和 L3.

1 在浓 $\mathrm{H}_{2} \mathrm{SO}_{4}$ 中进行磺化时, 由于 2 '位羟基的存在, 在 $90{ }^{\circ} \mathrm{C}$ 时, 硫酸容易磺化 $\mathbf{1}$ 的 $5^{\prime}$ 位, 生成一取代磺化物 L1. 在 $140{ }^{\circ} \mathrm{C}$ 时, 硫酸可以磺化 1 的 $2^{\prime}$ 位、5'位和 10 位, 生成三磺化物 $\mathbf{L 2}$. 在 90 和 $140{ }^{\circ} \mathrm{C}$ 之间主要生成的是 $\mathbf{L 1}$ 和 $\mathbf{L 2}$ 混合物(Scheme 3).
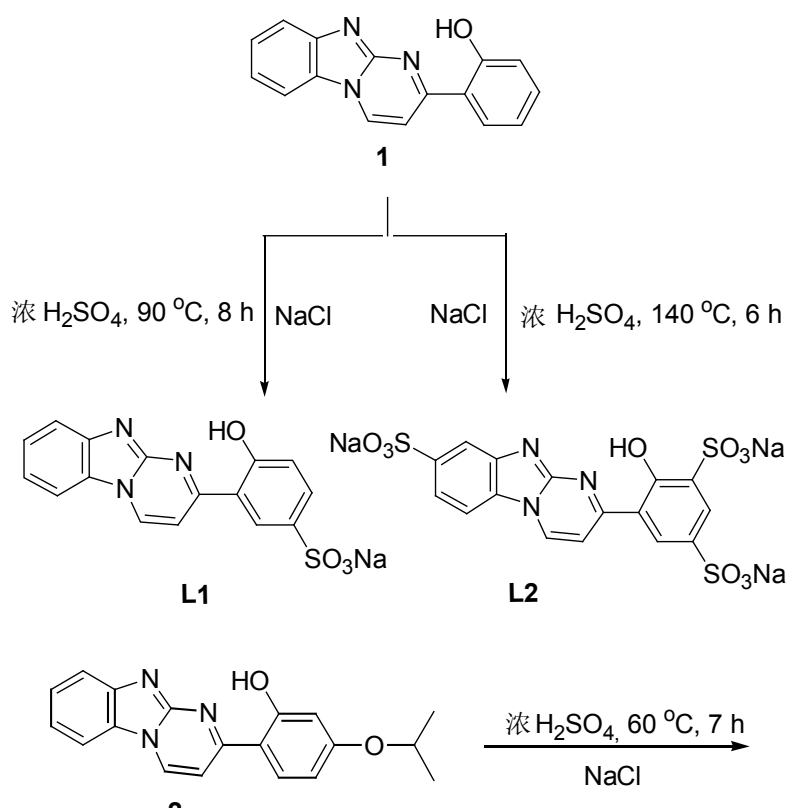

2<smiles>O=S(=O)(O)c1cc(-c2ccn3c(n2)nc2ccccc23)c(O)cc1O</smiles>

Scheme 3

2 在浓 $\mathrm{H}_{2} \mathrm{SO}_{4}$ 中进行磺化时, 4'位的异丙氧基被脱去 转变为羟基. 由于 $2^{\prime}$ 位和 4 '位双羟基的活化作用, 2 在 60 ${ }^{\circ} \mathrm{C}$ 时，硫酸就可以磺化 $5^{\prime}$ 位生成一取代磺化物 $\mathbf{L 3}$ (Scheme 3). 


\section{$2.2 \mathrm{~L} 1, \mathrm{~L} 2$ 和 $\mathrm{L} 3$ 的荧光光谱分析}

$\mathbf{L 1}$ 在水中溶解度比较低, 仅为 $1 \times 10^{-8} \mathrm{~mol} / \mathrm{L}$, 因此 将 $\mathbf{L 1}$ 配制成浓度为 $10 \mu \mathrm{mol} / \mathrm{L}$ 的二甲亚砜(DMSO)溶液. 将 $\mathbf{L 2}$ 和 $\mathbf{L 3}$ 配制成浓度为 $10 \mu \mathrm{mol} / \mathrm{L}$ 的水溶液. 苂光光 谱测定结果表明: L1、L2 和 L3 都具有明显的 ESIPT 效 应, Stokes 位移在 $175 \sim 225 \mathrm{~nm}$ 之间. 再分别向 L1, L2 和 $\mathbf{L 3}$ 的溶液中加入浓度为 $50 \mu \mathrm{mol} / \mathrm{L}$ 的 $\mathrm{Al}^{3+}$ 溶液. $\mathbf{L 1}$ 和 $\mathbf{L 3}$ 加入 $\mathrm{Al}^{3+}$ 后, 苂光光谱强度都明显减弱, 可能是 由于形成的 $\mathrm{Al}^{3+}$ 络合物分别不溶于 DMSO 和水. L1 的 激发峰和发射峰没有出现位移的变化; L3 的激发峰没 有位移变化, 但是发射峰由 $450 \mathrm{~nm}$ 红移到 $510 \mathrm{~nm}$. L2 加入 $\mathrm{Al}^{3+}$ 后, 苂光光谱强度增强约 20 倍, 激发峰由 350 $\mathrm{nm}$ 红移到 $405 \mathrm{~nm}$, 发射峰由 $520 \mathrm{~nm}$ 蓝移到 $505 \mathrm{~nm}$, 并 且 $\mathbf{L 2}$ 的苂光光谱对与 $\mathrm{Al}^{3+}$ 配位之后的光谱不存在干扰 作用(图 1), 因此 $\mathbf{L} \mathbf{2}$ 可以作为 $\mathrm{Al}^{3+}$ 的分子荧光探针.

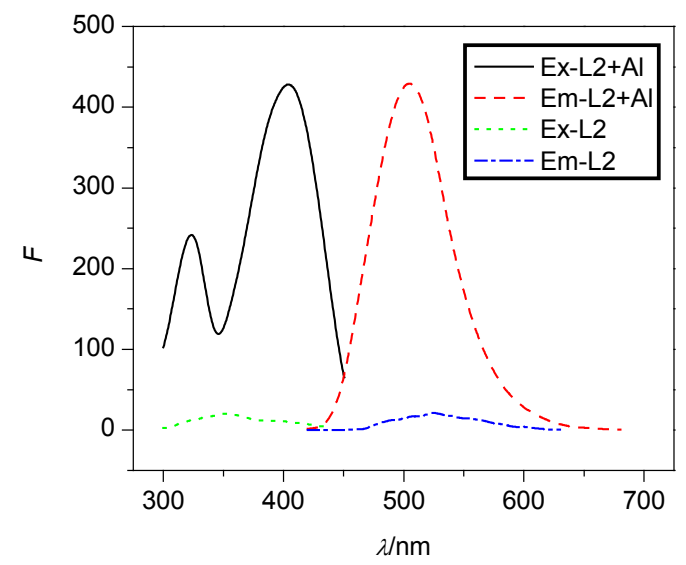

图 $1 \mathbf{L 2}(10 \mu \mathrm{mol} / \mathrm{L})$ 和 $\mathbf{L 2}(10 \mu \mathrm{mol} / \mathrm{L})$ 加 $\mathrm{Al}^{3+}(50 \mu \mathrm{mol} / \mathrm{L})$ 的 荧光光谱

Figure 1 Fluorescence spectrum of $\mathbf{L 2}(10 \mu \mathrm{mol} / \mathrm{L})$ and $\mathbf{L 2}(10$ $\mu \mathrm{mol} / \mathrm{L})$ with $\mathrm{Al}^{3+}(50 \mu \mathrm{mol} / \mathrm{L})$

\section{3 配体 L2 的光谱实验}

\subsection{1 配体 $\mathbf{L} 2$ 的荧光滴定}

将配体 $\mathbf{L} 2$ 配制成浓度为 $10 \mu \mathrm{mol} / \mathrm{L}$ 的水溶液，再分 别加入浓度为 $50 \mu \mathrm{mol} / \mathrm{L}$ 的金属离子 $\left(\mathrm{Al}^{3+}, \mathrm{Cu}^{2+}, \mathrm{Hg}^{2+}\right.$, $\left.\mathrm{K}^{+}, \mathrm{Mg}^{2+}, \mathrm{Na}^{+}, \mathrm{Ni}^{+}, \mathrm{Pb}^{2+}, \mathrm{Zn}^{2+}, \mathrm{Cr}^{3+}, \mathrm{Fe}^{3+}\right)$. 当激发波长 为 $405 \mathrm{~nm}$ 时, 荧光光谱在 $505 \mathrm{~nm}$ 处出现一个发射峰. 加入 $\mathrm{Cu}^{2+}, \mathrm{Hg}^{2+}, \mathrm{K}^{+}, \mathrm{Mg}^{2+}, \mathrm{Na}^{+}, \mathrm{Ni}^{2+}, \mathrm{Pb}^{2+}, \mathrm{Zn}^{2+}, \mathrm{Cr}^{3+}$, $\mathrm{Fe}^{3+}$, 发射峰的强度只有微弱的变化. 而加入 $\mathrm{Al}^{3+}$ 后, 荧光光谱发射峰的强度增加了约 20 倍. 表明配体 L2 对 其它金属离子响应很差, 对 $\mathrm{Al}^{3+}$ 具有专一的识别性(图 2).

为了进一步探究配体 $\mathbf{L} 2$ 与 $\mathrm{Al}^{3+}$ 的螯合方式, 做了 $\mathbf{L 2}$ 与 $\mathrm{Al}^{3+}$ 的菼光滴定实验. 依次将不同浓度的 $\mathrm{Al}^{3+}$ $(1 \sim 2.5$ equiv.)加入到浓度为 $10 \mu \mathrm{mol} / \mathrm{L}$ 的 $\mathbf{L 2}$ 中. 当

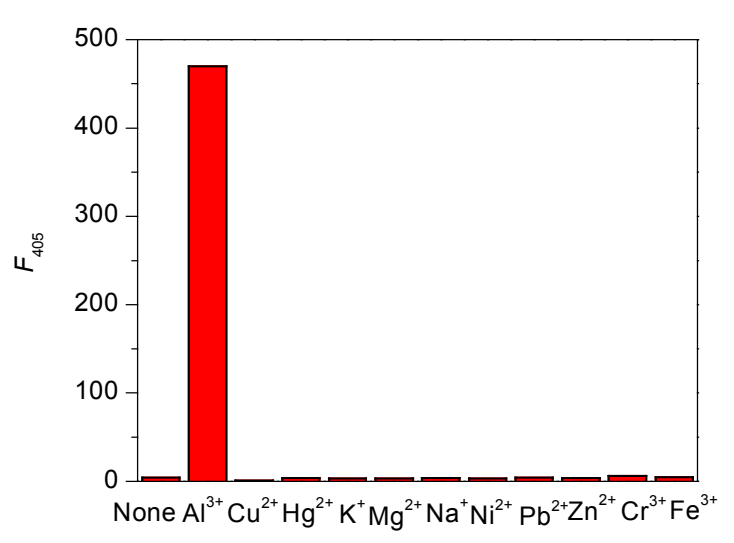

图 $2 \mathbf{L 2}(10 \mu \mathrm{mol} / \mathrm{L})$ 对不同的金属离子 $(50 \mu \mathrm{mol} / \mathrm{L})$ 的苂光响 应

Figure 2 The fluorescent response of $\mathbf{L 2}(10 \mu \mathrm{mol} / \mathrm{L})$ to different metal ions $(50 \mu \mathrm{mol} / \mathrm{L})$

$\mathrm{Al}^{3+}$ (1 2 equiv.)的浓度逐渐增加时, 可以发现 $505 \mathrm{~nm}$ 处的发射峰具有规律性的增强. 当 $\mathrm{Al}^{3+}$ 浓度为配体的 2 equiv. 的时候，菼光强度到达最大值. 随后，随 $\mathrm{Al}^{3+}$ 的浓 度增加(2.0 2.5 equiv.), 苂光强度不再增强, 表明 $\mathbf{L 2}$ 与 $\mathrm{Al}^{3+}$ 为 $1: 2$ 进行配合的(图 3).

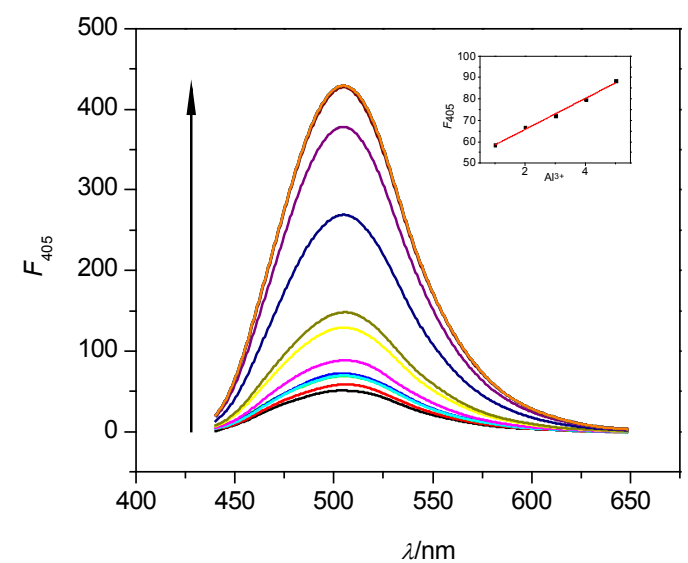

图 $3 \mathrm{L2}(10 \mu \mathrm{mol} / \mathrm{L})$ 随着 $\mathrm{Al}^{3+}$ 浓度增加 $(10 \sim 25 \mu \mathrm{mol} / \mathrm{L})$ 荧光 强度的变化

Figure 3 The emission intensity of $\mathbf{L 2}(10 \mu \mathrm{mol} / \mathrm{L})$ changes with increasing concentrations of $\mathrm{Al}^{3+}(10 \sim 25 \mu \mathrm{mol} / \mathrm{L})$

配体 $\mathbf{L 2}$ 与 $\mathrm{Al}^{3+}$ 的 Job's plot 曲线, 总浓度为 20 $\mu \mathrm{mol} / \mathrm{L}$. 从曲线可以观察到, 随着 $\mathrm{Al}^{3+}$ 所占的比例增大, 荧光强度随之增强，当 $\mathrm{Al}^{3+}$ 所占的比例为 0.7 的时候， 苂光强度最强，当 $\mathrm{Al}^{3+}$ 所占的比例继续增加时，荧光强 度逐渐变弱. 因此, 配体 $\mathbf{L 2}$ 与 $\mathrm{Al}^{3+}$ 为配合比 $1: 2$ (图 4).

最低检测限的测定, 先测定 $\mathbf{L 2}$ 的苂光强度 10 次, 然后计算 10 次测量的标准偏差 $(a)$. 之后在 $\mathbf{L 2}$ 的溶液中 滴加不同浓度的 $\mathrm{Al}^{3+}$, 根据测试结果找出线型范围, 在 这个线型关系下的斜率用 $k$ 表示. 其最低检测限等于 3 


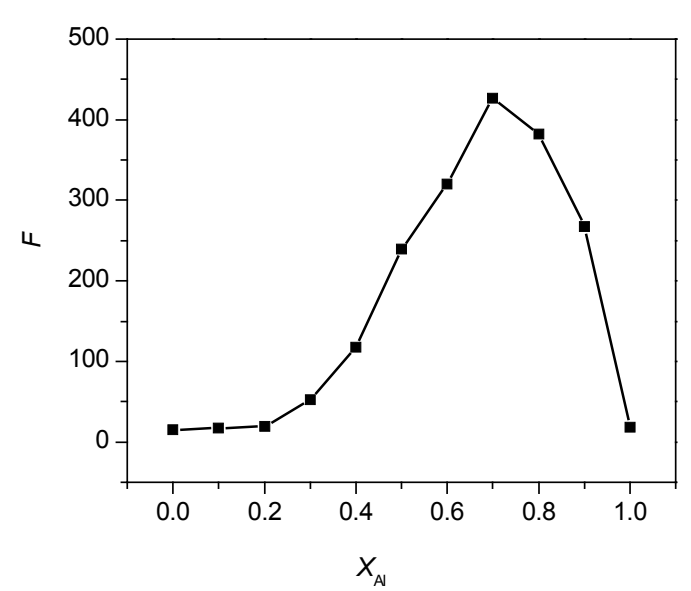

图 $4 \mathbf{L 2}$ 与 $\mathrm{Al}^{3+}$ 的 Job's plot $\left([\mathbf{L 2}]+\left[\mathrm{Al}^{3+}\right]=20 \mu \mathrm{mol} / \mathrm{L}\right)$ Figure 4 Job's plot of $\mathbf{L} 2$ and $\mathrm{Al}^{3+}\left([\mathbf{L 2}]+\left[\mathrm{Al}^{3+}\right]=20 \mu \mathrm{mol} / \mathrm{L}\right)$ 倍的标准偏差 $(3 a)$ 除以斜率 $k$, 即 $3 a / k^{[18]}$, 计算得到配体 $\mathbf{L 2}$ 对 $\mathrm{Al}^{3+}$ 的最低检测限为 $0.073 \mu \mathrm{mol} / \mathrm{L}$. 一般饮用水中 的 $\mathrm{Al}^{3+}$ 含量为 $0.05 \mathrm{mg} / \mathrm{L}^{3}$ (EPA standard) ${ }^{[18]}$, 因此该配 体可以用于人们日常生活中 $\mathrm{Al}^{3+}$ 的检测(图 3). 在波长 为 $365 \mathrm{~nm}$ 的紫外灯照射下, 向 $\mathbf{L 2}(10 \mu \mathrm{mol} / \mathrm{L})$ 的水溶液 中加入等量的 $\mathrm{Al}^{3+}$, 发现其荧光由无色变为绿色, 这种 明显的颜色变化, 表明 $\mathbf{L 2}$ 对 $\mathrm{Al}^{3+}$ 有良好的识别作用, 可 以实现对 $\mathrm{Al}^{3+}$ 的可视化检测.

\subsection{2 配体 $\mathrm{L} 2$ 的紫外滴定实验}

将配体 $\mathbf{L} 2$ 配制成浓度为 $100 \mu \mathrm{mol} / \mathrm{L}$ 的水溶液, 再 分别加入不同浓度的 $\mathrm{Al}^{3+}(0,100,200 \mu \mathrm{mol} / \mathrm{L})$, 测定紫 外吸收光谱. 结果表明, 随着 $\mathrm{Al}^{3+}$ 浓度的增加, $360 \mathrm{~nm}$ 处的吸收峰强度逐渐减弱, 可以说明 $\mathbf{L 2}$ 与 $\mathrm{Al}^{3+}$ 发生了 配位(图 5).

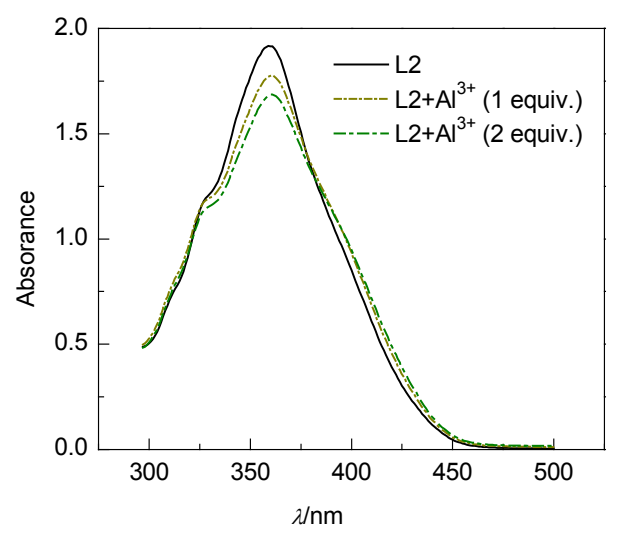

图 $5 \mathbf{L 2}(100 \mu \mathrm{mol} / \mathrm{L})$ 和不同浓度 $\mathrm{Al}^{3+}(0,100,200 \mu \mathrm{mol} / \mathrm{L})$ 的 紫外光谱

Figure 5 Changes of the UV-Vis spectra of $\mathbf{L 2}(100 \mu \mathrm{mol} / \mathrm{L})$ with externally added $\mathrm{Al}^{3+}(0,100,200 \mu \mathrm{mol} / \mathrm{L})$

\subsection{3 离子的竞争实验}

$\mathrm{Al}^{3+}(50 \mu \mathrm{mol} / \mathrm{L})$ 加入到含有配体 $\mathbf{L} 2(10 \mu \mathrm{mol} / \mathrm{L})$ 的
$\mathrm{Cu}^{2+}, \mathrm{K}^{+}, \mathrm{Mg}^{2+}, \mathrm{Na}^{+}, \mathrm{Ni}^{2+}, \mathrm{Pb}^{2+}, \mathrm{Zn}^{2+}, \mathrm{Cr}^{3+}, \mathrm{Fe}^{3+}(50$ $\mu \mathrm{mol} / \mathrm{L}$ ) 溶液中, 苂光光谱强度与单独加入 $\mathrm{Al}^{3+}$ 时的强 度无太大变化, 因此这些离子 $\left(\mathrm{Cu}^{2+}, \mathrm{K}^{+}, \mathrm{Mg}^{2+}, \mathrm{Na}^{+}\right.$, $\left.\mathrm{Ni}^{2+}, \mathrm{Pb}^{2+}, \mathrm{Zn}^{2+}, \mathrm{Cr}^{3+}, \mathrm{Fe}^{3+}\right)$ 对 $\mathbf{L} \mathbf{2}$ 与 $\mathrm{Al}^{3+}$ 的配位并无太 大的影响. 但是加入 $\mathrm{Hg}^{2+}$ 之后荧光强度明显的减弱, 因 此 $\mathrm{Hg}^{2+}$ 对 $\mathbf{L 2}$ 和 $\mathrm{Al}^{3+}$ 配位有影响, 可能是由于 $\mathrm{Hg}^{2+}$ 与配 合物中的 $\mathrm{Al}^{3+}$ 存在一些交换(图 6). 还研究了阴离子对 $\mathrm{Al}^{3+}$ 与 $\mathbf{L} \mathbf{2}$ 的配位之间的影响, 分别测定了 4 种阴离子不 同的铝盐 $\left[\mathrm{AlCl}_{3}, \mathrm{Al}\left(\mathrm{NO}_{3}\right)_{3}, \mathrm{Al}_{2}\left(\mathrm{SO}_{4}\right)_{3}, \mathrm{Al}\left(\mathrm{ClO}_{4}\right)_{3}\right]$ 与 $\mathbf{L} \mathbf{2}$ 的 荧光光谱, 4 种铝盐与 $\mathbf{L} \mathbf{2}$ 配位之后的荧光强度都近似相 等, 变化不大, 排除了阴离子对其配位的影响(图 7).

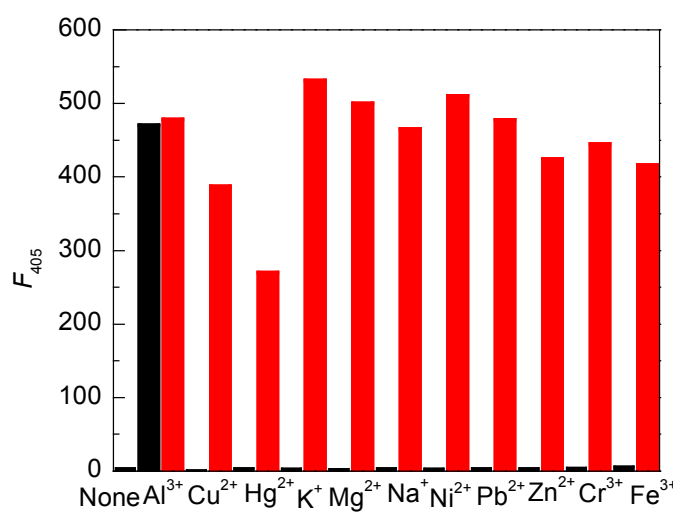

图 6 在各种金属离子 $(50 \mu \mathrm{mol} / \mathrm{L})$ 的存在下 $\mathrm{Al}^{3+}(50 \mu \mathrm{mol} / \mathrm{L})$ 对 $\mathbf{L 2}(10 \mu \mathrm{mol} / \mathrm{L})$ 荧光的影响

Figure 6 Effect of $\mathrm{Al}^{3+}(50 \mu \mathrm{mol} / \mathrm{L})$ on $\mathbf{L 2}(10 \mu \mathrm{mol} / \mathrm{L})$ in the presence of different metal ions $(50 \mu \mathrm{mol} / \mathrm{L})$

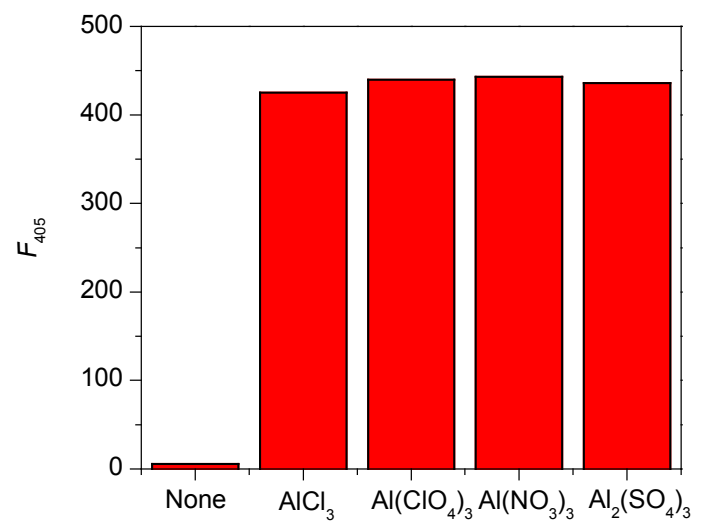

图 7 不同阴离子 $(50 \mu \mathrm{mol} / \mathrm{L})$ 对 $\mathbf{L 2}(10 \mu \mathrm{mol} / \mathrm{L})$ 与 $\mathrm{Al}^{3+}(50$ $\mu \mathrm{mol} / \mathrm{L}$ )荧光的影响

Figure 7 Effect of $\mathrm{Al}^{3+}(50 \mu \mathrm{mol} / \mathrm{L})$ on $\mathbf{L 2}(10 \mu \mathrm{mol} / \mathrm{L})$ in the presence of different anions $(50 \mu \mathrm{mol} / \mathrm{L})$

\subsection{4 配体与铝离子螯合机理探究}

为了了解 $\mathbf{L 2}$ 与 $\mathrm{Al}^{3+}$ 之间的配位方式, 本课题对 $\mathbf{L 2}$ 与 $\mathrm{Al}^{3+}$ 进行了核磁滴定实验. 分别在 $\mathbf{L 2}$ 的氛代 DMSO 溶液中加入 $0,1.0,2.0$ equiv. 的 $\mathrm{Al}^{3+}$, 当不加入 $\mathrm{Al}^{3+}$ 时, $\delta$ 12.3 处有一个峰, 属于 2 '位羟基; 当加入 1.0 equiv.的 
$\mathrm{Al}^{3+}$ 时, 峰面积明显减弱, 表明了 $\mathbf{L 2}$ 与 $\mathrm{Al}^{3+}$ 发生配位; 当加入 2 equiv. $\mathrm{Al}^{3+}$ 时, $2^{\prime}$ 位羟基的峰基本完全消失, 表 明了 $\mathbf{L 2}$ 与 $\mathrm{Al}^{3+}$ 以 $1: 2$ 的比例进行了配位(图 8).

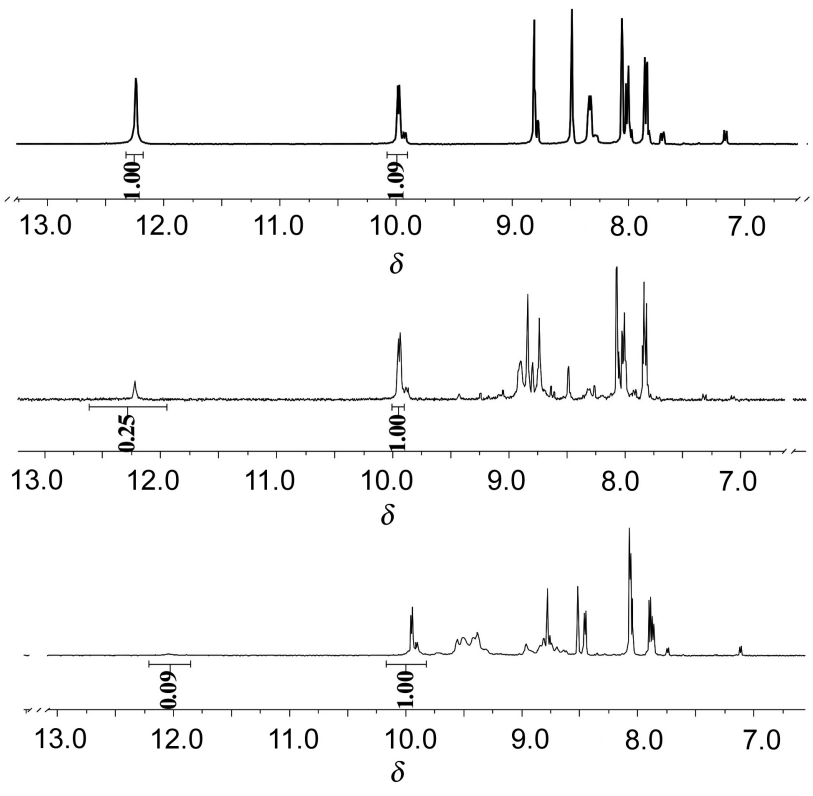

图 $8 \mathrm{L2}$ 和 $\mathrm{Al}^{3+}$ 的氢核磁滴定图谱

Figure $8{ }^{1} \mathrm{H}$ NMR titration experiments of $\mathbf{L} 2$ and $\mathrm{Al}^{3+}$

2 equiv. 的 $\mathrm{Al}^{3+}$ 与 $\mathbf{L} \mathbf{2}$ 进行配位时, 1 equiv. 的 $\mathrm{Al}^{3+}$ 会 与磺酸根上的 $\mathrm{Na}^{+}$进行交换, 另外 1 equiv. 的 $\mathrm{Al}^{3+}$ 与 $\mathbf{L 2}$ 进行配位(Scheme 4).

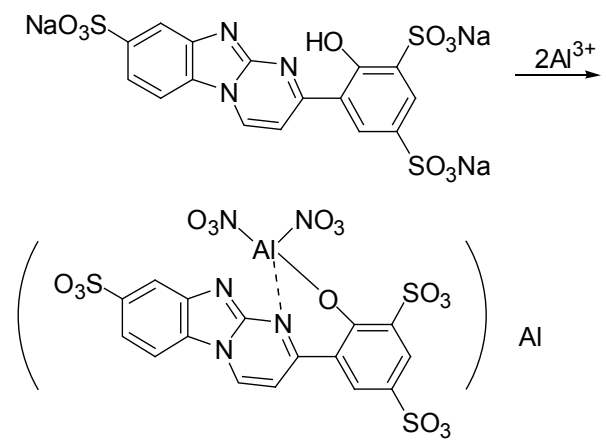

Scheme 4

\section{2 结论}

设计合成了 3 种嘧啶并 $[1,6-a$ 苯并咪唑磺化物 L1, $\mathbf{L 2}$ 和 $\mathbf{L 3}$, 由于 $\mathbf{L 1}$ 在水中溶解度低不能与 $\mathrm{Al}^{3+}$ 络合, $\mathbf{L 3}$ 在水中与 $\mathrm{Al}^{3+}$ 络合后荧光减弱, 因此 $\mathbf{L 1}$ 和 $\mathbf{L 3}$ 不适合作 为 $\mathrm{Al}^{3+}$ 的分子苂光探针. 苂光光谱实验表明: $\mathbf{L} 2$ 在水溶 液中能与 $\mathrm{Al}^{3+}$ 络合, 其在配位前存在激发态内分子质子 转移(ESIPT), 具有较大的 Stokes 位移 $(170 \mathrm{~nm})$, 当 $\mathbf{L 2}$ 与 $\mathrm{Al}^{3+}$ 进行配位之后, 荧光强度变强和 ESIPT 效应消 失, 并且 $\mathbf{L 2}$ 的苂光光谱对配位后的苂光光谱不存在影
响. $\mathbf{L 2}$ 对 $\mathrm{Al}^{3+}$ 具有很好的选择性和很高的灵敏度，可以 在水中对 $\mathrm{Al}^{3+}$ 进行分析和检测, 并且可用于可视化检测 $\mathrm{Al}^{3+}$, 在生物体和环境的监测中都具有广泛的适用性.

\section{3 实验部分}

\section{1 仪器与试剂}

X-5 显微熔点测定仪(控温型, 温度未经校正); ZF-2 型三用紫外仪; Bruker Tensor 27-IR 红外光谱仪( $\mathrm{KBr}$ 压 片法); BrukerDaltonics APEX(II) 4.7E FT-ICR Mass Spectrometer 型高分辨质谱仪; Bruker AM 400 超导傅立 叶数字化核磁共振仪 (TMS 为内标); 美国 PE 公司 PELS55 苂光分光光度计; 上海美谱达 UV-6100S 双光 束紫外/可见分光光度计.

邻羟基苯乙酮购于上海达瑞精细化工有限公司(纯 度 $>97 \%$ ), DMF-DMA 购于百灵威科技有限公司(纯度 $>98 \%$ )、二氨基苯并咪唑购于上海达瑞精细化工有限公 司(纯度 $>97 \%$ )、氢氧化钠、碳酸钾、石油醚、乙腈、 DMF、乙醇、乙酸乙酯等常用试剂均为分析纯，合成用 水为蒸馏水, 测试用水为去离子水, 各种金属的硝酸盐 均为 $\mathrm{AR}$ 级.

\section{2 实验方法}

\subsection{1 配体 $\mathbf{L 1}$ 和 $\mathbf{L} 2$ 的合成}

称取 $1 \mathrm{mmol}$ 的 $1(261.2 \mathrm{mg})$ 于 $5 \mathrm{~mL}$ 圆底烧瓶中, 加 入 $0.5 \mathrm{~mL}$ 浓硫酸. 在 $90{ }^{\circ} \mathrm{C}$ 下加热 $8 \mathrm{~h}, \mathrm{TLC}$ 检测, 待 $\mathbf{1}$ 消失后将反应液调至中性, 再倒入饱和氯化钠溶液中, 静置, 过滤, 得沉淀. 将沉淀用 $V(\mathrm{EtOH}): V\left(\mathrm{H}_{2} \mathrm{O}\right)=1$ : 1 的溶液重结晶, 得黄色沉淀 L1 (243.2 mg), 产率 67\%. 或在 $140{ }^{\circ} \mathrm{C}$ 下加热 $6 \mathrm{~h}, \mathrm{TLC}$ 检测, 待 $\mathbf{1}$ 消失后将反应液 调至中性, 再倒入饱和氯化钠溶液中, 静置, 过滤, 得 沉淀. 再用 $20 \%$ 的氯化钠水溶液重结晶 3 次, 得黄色沉 淀 L2 (368.8 mg), 产率为 65\%.

\subsection{2 配体 $\mathbf{L 3}$ 的合成}

称取 $1 \mathrm{mmol}$ 的 $2(319.4 \mathrm{mg})$ 的于 $5 \mathrm{~mL}$ 圆底烧瓶中, 加入 $0.5 \mathrm{~mL}$ 浓硫酸, 在 $60{ }^{\circ} \mathrm{C}$ 下, 加热回流 $7 \mathrm{~h}, \mathrm{TLC}$ 监 测, 待 2 消失后, 将反应液调至中性, 倒入饱和氯化钠 溶液中, 静置, 过滤, 得沉淀. 将沉淀用 $V(\mathrm{EtOH})$ : $V\left(\mathrm{H}_{2} \mathrm{O}\right)=1: 1$ 的溶液重结晶, 得黄色沉淀 $\mathbf{L 3}$ (273.1 $\mathrm{mg}$ ), 产率为 $72 \%$.

4-(2'-羟基苯基)嘧啶并 $[1,6-a$ 苯并咪唑-5'-磺酸钠 (L1): 黄色固体. m.p. $>320{ }^{\circ} \mathrm{C}$ (decomposed); ${ }^{1} \mathrm{H}$ NMR $\left(400 \mathrm{MHz}\right.$, DMSO- $\left.d_{6}\right) \delta: 11.69(\mathrm{~s}, 1 \mathrm{H}), 9.84(\mathrm{~d}, J=7.3 \mathrm{~Hz}$, $1 \mathrm{H}), 8.85 \sim 8.18(\mathrm{~m}, 3 \mathrm{H}), 7.91(\mathrm{~d}, J=8.1 \mathrm{~Hz}, 1 \mathrm{H}), 7.81(\mathrm{t}$, $J=7.7 \mathrm{~Hz}, 1 \mathrm{H}), 7.77 \sim 7.64(\mathrm{~m}, 2 \mathrm{H}), 7.08(\mathrm{~d}, J=8.5 \mathrm{~Hz}$, $1 \mathrm{H}) ;{ }^{13} \mathrm{C}$ NMR $\left(101 \mathrm{MHz}, \mathrm{CF}_{3} \mathrm{COOH}\right) \delta: 163.9,159.9$, 
$140.6,133.2,131.1,130.8,128.0,126.78,125.3,123.6$, $121.7,116.9,110.69,109.21$; IR (KBr) v: 3685, 3498, 3412, 3027, 1714, 1553, 1491, 1404, 1332, 1278, 1210, $1177,819,763,700,566 \mathrm{~cm}^{-1}$. HRMS calcd for $\mathrm{C}_{16} \mathrm{H}_{10^{-}}$ $\mathrm{N}_{3} \mathrm{O}_{4} \mathrm{~S}[\mathrm{M}-\mathrm{Na}]^{-}$340.0392, found 340.0371.

4-(2'-着基苯基)嘧啶并 $[1,6-a$ 苯并咪唑-3',5',10-三磺 酸钠(L2): 黄色固体. m.p. $>320{ }^{\circ} \mathrm{C}$ (decomposed); ${ }^{1} \mathrm{H}$ NMR (400 MHz, DMSO-d $\left.d_{6}\right) \delta: 12.24(\mathrm{~s}, 1 \mathrm{H}), 9.98$ (d, $J=$ $7.1 \mathrm{~Hz}, 1 \mathrm{H}), 8.82(\mathrm{~s}, 1 \mathrm{H}), 8.49(\mathrm{~s}, 1 \mathrm{H}), 8.34(\mathrm{~d}, J=6.8 \mathrm{~Hz}$, $1 \mathrm{H}), 8.16 \sim 7.98(\mathrm{~m}, 2 \mathrm{H}), 7.85(\mathrm{~d}, J=8.3 \mathrm{~Hz}, 1 \mathrm{H}) ;{ }^{13} \mathrm{C}$ NMR (101 MHz, DMSO- $\left.d_{6}\right) \delta: 169.8,164.8,154.1,104.7$, $144.8,139.4,137.7,131.6,129.6,127.1,124.6,121.5$, $113.4,112.5,111.45$; IR $v: 3687,3027,1718,1552,1492$, $1445,1404,1333,1276,1210,1181,1106,810,764,701$ $\mathrm{cm}^{-1}$; HRMS calcd for $\mathrm{C}_{16} \mathrm{H}_{8} \mathrm{~N}_{3} \mathrm{O}_{10} \mathrm{~S}_{3}[(\mathrm{M}-3 \mathrm{Na}) / 3]^{-}$: 165.9790, found 165.9795 .

4-(2',4'-二美基苯基)嘧啶并 $[1,6-a$ ]苯并咪唑-5'-磺酸 钠 (L3): 黄色固体. m.p. $>320{ }^{\circ} \mathrm{C}$ (decomposed); ${ }^{1} \mathrm{H}$ NMR (400 MHz, DMSO- $\left.d_{6}\right) \delta: 13.07(\mathrm{~s}, 1 \mathrm{H}), 11.21(\mathrm{~s}$, $1 \mathrm{H}), 9.59$ (d, $J=7.4 \mathrm{~Hz}, 1 \mathrm{H}), 8.50 \sim 8.23(\mathrm{~m}, 2 \mathrm{H}), 8.06$ (d, $J=7.4 \mathrm{~Hz}, 1 \mathrm{H}), 7.83(\mathrm{~d}, J=8.1 \mathrm{~Hz}, 1 \mathrm{H}), 7.63(\mathrm{t}, J=7.7$ $\mathrm{Hz}, 1 \mathrm{H}), 7.52$ (t, $J=7.7 \mathrm{~Hz}, 1 \mathrm{H}), 6.43(\mathrm{~s}, 1 \mathrm{H}) ;{ }^{13} \mathrm{C}$ NMR $\left(101 \mathrm{MHz}, \mathrm{DMSO}-d_{6}\right) \delta: 163.0,162.4,158.9,147.2,136.6$, $129.4,127.3,126.6,124.7,122.5,116.7,112.8,111.2$, 106.4, 103.7; IR (KBr) v: 3685, 3498, 3413, 3027, 1715, 1552, 1491, 1446, 1404, 1333, 1277, 1210, 1178, 819, 763, $701,566 \mathrm{~cm}^{-1}$; HRMS calcd. for $\mathrm{C}_{16} \mathrm{H}_{10} \mathrm{~N}_{3} \mathrm{O}_{5} \mathrm{~S}[\mathrm{M}-\mathrm{Na}]^{-}$: 356.0341 , found 356.0329 .
辅助材料(Supporting Information) 提供了各化合物 的苂光光谱、氢谱、碳谱以及质谱数据. 这些材料可以 免费从本刊网站(http://sioc-journal.cn/)上下载.

\section{References}

[1] Atkins, J. G. M.; Burgess, E. M. J. Am. Chem. Soc. 1968, 17, 4744.

[2] Srinivasan, P. T.; Viraraghavan, T.; Subramanian, K. S. Water Sa. 1999, $25,47$.

[3] Delhaize, E.; Ryan, P. R. J. Plant Physiol. 1995, 107, 315.

[4] Godbold, D. L.; Fritz, E.; Hüttermann, A. Proc. Natl. Acad. Sci. U. S. A. 1988, 11, 3888 .

[5] Ren, J.; Tian, H. Sensors 2007, 12, 3166.

[6] Burwen, D. R.; Olsen, S. M.; Bland, L. A.; Arduino, M. J.; Reid, M. H.; Jarvis, W. R. Kidney. Int. 1995, 2.

[7] Fasman, G. D. Coord. Chem. Rev. 1996, 149, 125.

[8] Nayak, P. Environ. Res. 2002, 89, 101.

[9] Cronan, C. S.; Walker, W. J.; Bloom, P. R. Nature 1986, 6093, 140.

[10] Ding, Y.; Li, X.; Li, T.; Zhu, W.-H.; Xie, Y.-S. J. Org. Chem. 2013, $78,5328$.

[11] Li, P.; Zeng, Y.; Chen, J. P.; Li, Y. Y.; Li, Y. Acta Chim. Sinica 2012, 70, 1611 (in Chinese).

(李鹏, 曾毅, 陈金平, 李迎迎, 李嫕, 化学学报, 2012, 70, 1611.)

[12] Berthon, G. Coord. Chem. Rev. 2002, 228, 319

[13] Bielarczy, K. H.; Jankowska, A.; Madziar, B.; Matecki, A.; Michno, A.; Szutowicz, A. Neurochem. Int. 2003, 42, 323.

[14] Yousef, M. I.; El-Morsy, A.; Hassan, M. S. Toxicology 2005, 215, 97.

[15] Sen, S.; Mukherjee, T.; Chattopadhyay, B.; Moirangthem, A.; Basu, A.; Marek, J.; Chattopadhyay, P. Analyst 2012, 17.

[16] Zhou, Y.; Zhang, J.; Zhou.; Zhang, L.; Zhang, M. Spectrochim. Acta, $A$ 2013, 106, 68.

[17] Liu, L. Z. M.S. Thesis, Shaanxi Normal University, Xi'an 2013, p. 41

(刘龙珠, 硕士论文, 陕西师范大学, 西安, 2013, p. 41.)

[18] Wang, L. N.; Qin, W. W.; Tang, X. L.; Dou, W.; Liu, W.; Teng, Q.; Yao, X. Org. Biomol. Chem. 2010, 16, 3751. 